\title{
Encapsulement humoral \\ en tant que réaction de défense chez les Diptères
}

\author{
par P. GOETZ, I. ROETTGEN et W. LINGG \\ Biologisches Institut I der Universität, Katharinenstr. 20, D-78 Freiburg (R.F.A.)
}

Chez beaucoup d'Invertébrés, la réaction de défense classique envers les parasites: métazoaires est l'encapsulement cellulaire, c'est-à-dire la formation autour du parasite d'une enveloppe d'hémocytes qui empêche son développement. Une variation de cette réaction, un encapsulement humoral, fut d'abord décrit par Bronskill (1962) et Esslinger (1962) pour les Culicidés envers des Nématodes parasites. Les auteurs cités rapportent la précipitation d'une substance brunâtre sur la surface des Nématodes qui se produisait sans participation visible des hémocytes.

Pour une étude de cette réaction humorale, les larves des Chironomides (Diptera) étaient particulièrement utiles. Depuis notre première étude (Goetz, 1969), nous avons obtenu les résultats suivants :

L'encapsulement humoral est une réaction efficace de défense envers les Mermithides, Nématodes parasites de Chironomus. Le fait qu'il a lieu in vitro dans l'hémolymphe isolée permet son observation directe sous le microscope et la manipulation des conditions expérimentales: par exemple, l'addition des inhibiteurs enzymatiques. La précipitation de la substance capsulaire commence après 2-5 minutes ; environ 10 minutes plus tard, l'enveloppe est complète (Goetz, 1973);

L'encapsulement humoral a lieu non seulement envers des Nématodes mais aussi envers d'autres pathogènes comme les bactéries (Bacillus thuringiensis, Pseudomonas aeruginosa, Serratia marcescens), des champignons (Beauveria bassiana, Mucor hiemalis, Aspergillus niger) et des microsporidies (Mrazekia brevicauda). Parmi les matériaux śtrangers (non-vivants), il y a des substances provocatrices et d'autres qui ne provoquent pas un encapsulement humoral. Introduit dans l'hémocoele de Chironomus ou mis en contact avec l'hémolymphe isolée, des matériaux comme le Sephadex (polysaccharide), le Latex (polystyrène) et l'agar sont encapsulés alors que des morceaux de cellophane, 
de nylon, du fil de verre, de la poudre de fer, etc... ne provoquent aucune réaction visible (Goetz, 1973).

Quant à la nature chimique de la réaction humorale, nous avons donné (Goetz et Vey, 1974 ; Vey et Goetz, 1975) des preuves histochimiques, enzymatiques et physiolo-

TABleau I. - Type d'encapsulement (humoral ou cellulaire) chez les insectes

\begin{tabular}{|c|c|c|c|c|}
\hline & & humoral & cellulaire & $\begin{array}{l}\text { nombre } \\
\text { d'hémocytes } \\
\text { per } \mathrm{mm}^{3}\end{array}$ \\
\hline Diptera $\ldots \ldots \ldots \ldots \ldots$ & $\begin{array}{l}\text { Chironomus } \\
\text { Endochironomus } \\
\text { Glyptotendipes } \\
\text { Corethra } \\
\text { Culex } \\
\text { Psychoda } \\
\text { Erystalomya } \\
\text { Microchrysa } \\
\text { Simulium } \\
\text { Liponeura } \\
\text { Tabanus } \\
\text { Calliphora }\end{array}$ & $\begin{array}{l}+ \\
+ \\
+ \\
+ \\
+ \\
+ \\
+ \\
+ \\
- \\
-\end{array}$ & $\begin{array}{l}- \\
z \\
- \\
z \\
- \\
+ \\
+ \\
+ \\
+\end{array}$ & $\begin{array}{r}2000 \\
2500 \\
1000 \\
500 \\
4000 \\
3000 \\
1500 \\
1000 \\
50000 \\
16000 \\
8000 \\
26000\end{array}$ \\
\hline Ephemeroptera $\ldots \ldots \ldots$ & $\begin{array}{l}\text { Ephemera } \\
\text { Epeorus }\end{array}$ & - & $\stackrel{+}{+}$ & $\begin{array}{l}\text { (1) } \\
\text { (1) }\end{array}$ \\
\hline $\begin{array}{l}\text { Plecoptera } \quad \ldots \ldots \ldots \ldots \\
\text { Odonata } \ldots \ldots \ldots \ldots \\
\text { Blattoidea } \\
\text { Orthoptera } \\
\text { Orth } \ldots \ldots \ldots\end{array}$ & $\begin{array}{l}\text { Perla } \\
\text { (Anisoptera) } \\
\text { Periplaneta } \\
\text { Gryllotalpa }\end{array}$ & $\begin{array}{l}- \\
-\end{array}$ & $\begin{array}{l}+ \\
+ \\
+ \\
+\end{array}$ & $\begin{array}{c}(1) \\
(1) \\
30000 \\
(1)\end{array}$ \\
\hline Heteroptera $\ldots \ldots \ldots \ldots$ & $\begin{array}{l}\text { Ranatra } \\
\text { Corixa }\end{array}$ & - & $\begin{array}{l}+ \\
+\end{array}$ & $\begin{array}{l}6000 \\
(1)\end{array}$ \\
\hline Megaloptera $\quad . \ldots \ldots \ldots$ & Sialis & 一 & + & 20000 \\
\hline Coleoptera $\ldots \ldots \ldots \ldots$ & $\begin{array}{l}\text { Dytiscus } \\
\text { Tenebrio }\end{array}$ & - & + & $\begin{array}{l}10000 \\
30000\end{array}$ \\
\hline Trichoptera $\quad \ldots \ldots \ldots \ldots$ & $\begin{array}{l}\text { Hydropsyche } \\
\text { Rhyacophila } \\
\text { Stenophylax }\end{array}$ & E & $\begin{array}{l}+ \\
+ \\
+\end{array}$ & $\begin{array}{r}7000 \\
30000 \\
25000\end{array}$ \\
\hline Lepidoptera $\ldots \ldots \ldots \ldots$ & Galleria & 一 & + & 60000 \\
\hline
\end{tabular}

(1) Nombre d'hémocytes encore à déterminer.

giques que la substance capsulaire doit être un complexe protéine-polyphénol. Les deux réactions, l'encapsulement cellulaire et l'encapsulement humoral donnent le même résultat. Dans le cas de l'encapsulement cellulaire, il faut distinguer entre la formation de l'enveloppe cellulaire comme premier pas et la production d'une substance capsulaire par les cellules intérieures de l'enveloppe qui finit par le dépôt d'une couche mélanisée à la surface du parasite ou du corps étranger (Poinar et al., 1968). Le principal de l'encapsulement est la formation d'une couche résistante autour du parasite qui cause -sa mort en empêchant l'échange d'oxygène et de substances nutritives. 
En dehors de l'hémocoele, l'encapsulement humoral se réalise aussi dans la cuticule (envers les filaments du champignon Beauveria bassiana). L'enzyme phenoloxidase, que nous rendons responsable de l'encapsulement humoral fut mis en évidence par Maier (1973), dans l'hémolymphe, certains hémocytes (les granulocytes) et dans la cuticule des larves de Chironomus.

Lors de recherches récentes (Goetz et Lingg, en préparation), nous avons essayé de mettre en évidence l'existence de l'encapsulement humoral chez d'autres insectes. Des représentants de onze ordres d'insectes furent contrôlés en utilisant des Nématodes, des champignons, du Sephadex et de l'agar comme agents provocateurs. La plupart des insectes contrôlés montraient un encapsulement cellulaire; seul, parmi les Diptères, nous avons trouvé plusieurs espèces avec un encapsulement humoral (tableau I). Le type de la réaction est en corrélation avec l'image sanguine: le nombre d'hémocytes est nettement moins élevé chez les larves des Diptères à encapsulement humoral, ils ont en moyenne $2000(500-4000)$ cellules $/ \mathrm{mm}^{3}$, tandis qu'on trouve 24000 (7000 40000 ) dans le groupe à réaction cellulaire.

\section{Bibliographie}

Bronskill (J.F.), 1962. - Encapsulation of Rhabdidoid nematodes in mosquitoes. Canad. J. Zool., 40, 1269-1275.

Esslinger (J. H.), 1962. - Behaviour of microfilaria of Brugia pahangi in Anopheles quadrimaculatus. Amer. J. Trop. Med. Hyg., 11, 749.

Goetz (P.), 1969. - Die Einkapselung von Parasiten in der Haemolymphe von Chironomus-Larven (Diptera). Zool. Anz., Suppl., 33, 610-617.

GoETZ (P.), 1973. - Immunreaktionen bei Insekten. Naturw. Rdsch., 26, 367-375.

GoETZ (P.), 1974. - Humorale Einkapselung von Hydromermis contorta und Turbatrix aceti (Nematoda) in Haemolymphe von Chironomus thummi (Diptera). Publ. wiss. Film, Sekt. Biologie, 7, 67-79.

GoETZ (P.) et VEY (A.), 1974. - Humoral encapsulation in Diptera (insecta) : defence reactions of Chironomus larvae against fungi. Parasitology, 68, 193-205.

Goetz (P.) et Lingg (W.) (en préparation).

Maier (W. A.), 1973. - Die Phenoloxydase von Chironomus thummi und ihre Beeinflussung durch parasitäre Mermithiden. J. Insect Physiol., 19, 85-95.

Poinar (G. O. jr.), Leutenegger (R.) et Goetz (P.), 1968. - Ultrastructure of the formation of a melanotic capsule in Diabrotica (Coleoptera) in response to a parasitic nematode (Mermithidae). J. Ultrastruct. Res., 25, 293-306.

VeY (A.) et Goetz (P.), 1975. - Humoral encapsulation in Diptera (Insecta) : comparative studies in vitro. Parasitology, 70, 77-86. 